\title{
Control Over a Network: Using Actuation Buffers to Reduce Transmission Frequency
}

\author{
Michael Epstein, Ling Shi, Stefano Di Cairano and Richard M. Murray
}

\begin{abstract}
We consider a discrete time linear feedback control system with additive noise where the control signals are sent across a network from the controller to the actuators. Due to network considerations it is desired to reduce the transmission frequency of the control signals. We show that by including a finite sequence of predicted control signals in each communication packet the frequency of transmission can be reduced by transmitting only when the previously sent sequence has run out, although as a consequence the closed loop error will increase. We introduce a communication protocol, which we call Input Difference Transmission Scheme (IDTS), that transmits control packets when the difference between newly computed control values and the predicted control sequence previously transmitted is larger than a certain threshold. This threshold is a design parameter and we show how the closed loop behavior varies with this threshold. Simulation results are provided to augment the theory.
\end{abstract}

\section{INTRODUCTION}

The use of communication networks in feedback loops, termed Networked Control Systems (NCS) [1], has become a fast growing area of research. There are several advantages for introducing communication networks in feedback loops, including modularity and reconfigurability of system components, however potential issues arise when closing the loop around imperfect communication links including data dropout, delays and quantization effects. Researchers have addressed many of these issues, see [2] for a recent survey.

Often the information communicated in NCS is in the form of packets [3]. These packets have a fixed header length and additional space to be used for data. Due to the overhead included in each transmission, if the frequency of transmission is decreased there will be a corresponding savings in bandwidth and decrease in network congestion. Thus it is desired to send fewer but more informative packets. In this work we are concerned with how much extra information should be included in the packets, when they should be transmitted and the impacts on closed loop performance.

In this work we assume control values are transmitted across a communication link to the plant, a schematic of this situation is shown in Fig. 1. A motivating example for this type of system is those similar to [4] where remote vehicles with limited computation are sent trajectory and/or control commands from remote processing units. To make the packets more informative we will include not only the control value computed for the current time step but also predicted control values for the next $N$ steps in the future.

Michael Epstein, Ling Shi and Richard Murray are all in the Division of Engineering and Applied Science at the California Institute of Technology. Stefano di Cairano is a $\mathrm{PhD}$ student at the University of Sienna in Italy. epstein, shiling, cairano, murraydcds.caltech.edu
We will investigate the closed loop performance as a function of this buffer length under different communication schemes. First we consider the case that the controller transmits only when the buffer at the actuator is empty. We will also introduce a communication protocol, which we call the Input Difference Transmission Scheme (IDTS), that calculates a new control sequence at every time step but only transmits to the actuator when the difference between the new sequence and that in the buffer has exceeded a threshold. We will show how this scheme can improve the closed loop performance and provide more flexibility for the system designer.

Other researchers have studied ways to determine access to a shared communication medium by nodes of actuators and sensors. In [5] they provide conditions for a stabilizing communication sequence to exist and an algorithm to construct such a sequence. This uses a static scheduling protocol and assumes zero values when transmission does not occur rather than incorporating some form of an estimator or buffer. A similar setting is considered in [6] but they include estimators on the receiving side of every network transmission to estimate the value of a signal when it is not transmitted. In addition to a static transmission policy they study granting access to the nodes whose difference between estimated and true signal is largest. The problem setting is slightly different from the one considered here since they consider continuous plants and are not concerned with limiting transmission frequency. In [7] an optimal communication logic is developed to strike a balance between closed loop error and communication rate. The logic that results is similar to the IDTS in this paper, data will only transmit when an estimated state differs from a true state by more than a specified amount, however they transmit state information rather than control values and thus make no use of a control buffer.

The notion of using a buffer with predicted control values in a NCS setting is not necessarily new, for example see [8], [9], however not much has been investigated relating the length of the buffer to the closed loop performance, especially in terms of reducing the communication frequency. In [10] the authors consider a similar setting, but their analysis of performance as a function of the buffer size is affected by the discretization sampling time of a continuous time plant. Furthermore, they have not considered the effect on the transmission frequency. In [11] the authors consider transmitting a packet that contains future control signals, but they are not concerned with limiting the transmission frequency, only reducing the effect of communication losses.

The remainder of the paper is organized as follows. In 
Section II a mathematical description of the problem setting is given. The communication protocol is introduced and analyzed in Section III. Simulation examples are shown in Section IV. Finally the paper concludes with a summary of the work and future directions in Section V.

\section{Problem Set UP}

We consider discrete time linear time invariant systems of the form

$$
x_{k+1}=A x_{k}+B u_{k}+w_{k}
$$

where $x_{k} \in \mathbb{R}^{n}$ is the state, $u_{k} \in \mathbb{R}^{r}$ is the control input and $w_{k} \in \mathbb{R}^{n}$ is an unknown but bounded disturbance. We also assume a bound on the initial condition so that

$$
\left\|w_{k}\right\| \leq \bar{W} \forall k \text { and }\left\|x_{0}\right\| \leq \chi .
$$

Unless otherwise stated the norm $\|\cdot\|$ will be the two-norm for vectors and the induced two-norm for matrices.

Further we assume $A$ is unstable, the pair $(A, B)$ is controllable and that a feedback gain $F$ has been designed so that in the absence of the network the control signal would be $u_{k}=F x_{k}$ and $(A+B F)$ is stable. We consider the case where the control signal $u_{k}$ arriving at the actuators/plant is transmitted across a network from a remotely located controller, as shown in Fig. 1. We will ignore delay, quantization and lost information effects of the network.

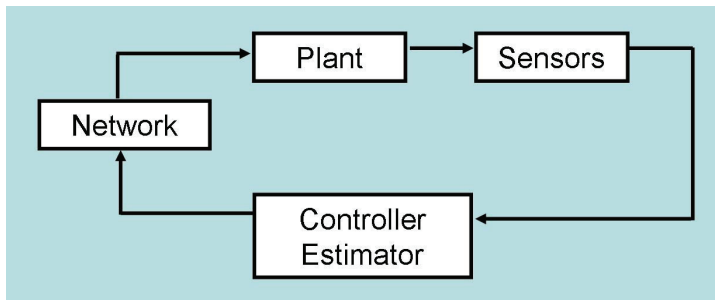

Fig. 1. NCS feedback loop.

The information is transmitted in a packet containing a fixed amount of overhead. As discussed earlier, it is advantageous to put more information into a single packet and reduce the frequency of transmission. We use an anticipative controller that will transmit a sequence of control steps each time a packet is sent to plant. In [12] the authors use an anticipative continuous-time controller as a way to reduce the negative effects introduced by network delays, while our aim is to use it to reduce transmission frequency. In addition we propose different methods for determining when to transmit the packets. The overall performance of the system we consider is the closed loop error and the frequency of transmission of the control packets, with a desire to keep both of these quantities low. The tradeoff is that lowering the transmission frequency can increase the error. We will analyze how the closed loop performance varies with the length of the control sequences transmitted and by using different transmission protocol.

At every instance in time a control signal for the current time and any future time can be computed based on the current state. Denote the control signal to be applied at time $k+j$ but computed at time $k$ by $u_{k+j \mid k}, j=0,1, \ldots$ Note the information available to the controller when calculating $u_{k+j \mid k}$ is $\mathcal{I}_{k}=\left\{\mathcal{I}_{k-1}, x_{k}, u_{k-1}\right\}$. We consider a controller that at every time instant computes a control signal for the current time and $N \geq 0$ time steps in the future, i.e. $\left\{u_{k \mid k}, u_{k+1 \mid k}, \ldots, u_{k+N \mid k}\right\}$. The information packet that can be transmitted from the controller to the plant at time $k$ is exactly this control sequence

$$
\mathbf{U}_{k}=\left\{u_{k \mid k}, u_{k+1 \mid k}, \ldots, u_{k+N \mid k}\right\} .
$$

Denote the elements of the packet by $\mathbf{U}_{k}(j)=u_{k+j-1 \mid k}$, $j=1,2, \ldots, N+1$. With this scheme each control packet contains $r \cdot(N+1)$ data points.

When the plant receives packet $\mathbf{U}_{k}$ it discards all previously buffered commands and follows the current control sequence. If a packet is not received the plant applies the corresponding control signal in the buffer from the last previously received packet. For example, assume that at time $k+M$ the last previously received packet was that from time $k$, then the control signal applied to the plant would be $u_{k+M}=u_{k+M \mid k}=\mathbf{U}_{k}(M+1)$. Since we have assumed a finite packet length, $N<\infty$, we must decide what to do when $M>N$. In this case the last previously received packet will only contain control signals up to time $u_{k+N \mid k}$, so we must choose what control signal the plant will apply for time $u_{k+N+j}, j=1,2, \ldots$. There are two possibilities: apply zero control, $u_{k+N+j}=0$, or hold the control from the last command in the sequence, $u_{k+N+j}=u_{k+N \mid k}$.

The control applied to the plant at time $k+M$ assuming the last transmitted packet was sent at time $k$ is

$$
u_{k+M}=\left\{\begin{array}{c}
u_{k+M \mid k}=\mathbf{U}_{k}(M+1) \text { if } M \leq N \\
\gamma u_{k+N \mid k}=\gamma \mathbf{U}_{k}(N+1) \text { otherwise }
\end{array}\right.
$$

where $\gamma \in\{0,1\}$ indicates if the choice is to use zero control $(\gamma=0)$ or hold the last command $(\gamma=1)$. If a state feedback controller is used with the future controls signals based on the predicted evolution of the system, then we see

$$
\mathbf{U}_{k}=\left\{F x_{k}, F(A+B F) x_{k}, \ldots, F(A+B F)^{N} x_{k}\right\} .
$$

\section{Transmit Protocol}

To reduce the amount of traffic on the network, the controller will not transmit every control packet $\mathbf{U}_{k}$. There are several options for determining when to transmit the control packet, they are explored below.

\section{A. Fixed Transmission Time}

The simplest scheme to implement is that with a fixed transmission time. Given that the length of the control buffer is $N$, so that each control packet contains the current control signal and the next $N$ predicted control signals, if the packet is transmitted at time $k$ then the control buffer will not be empty until time $k+N+1$. Thus if the control sequence is transmitted every $N+1$ time steps the actuator will always have a control signal to apply. We transmit the first control packet $\mathbf{U}_{0}$, thus the packets that will be transmit are $\left\{\mathbf{U}_{0}, \mathbf{U}_{N+1}, \mathbf{U}_{2 \cdot(N+1)}, \ldots\right\}$. 
If the control sequence were transmitted more frequently there would be some elements of the transmitted control buffer that would never be implemented and these would be unnecessary to include. For example, if the packet that was sent at time $k$ contained $u_{k+N+j \mid k}, j=1,2, \ldots$ these control signals would never be applied since at time $k+$ $N+1$ a new packet is transmitted to the actuator containing $u_{k+N+1 \mid k+N+1}$ and this is applied to the plant. Thus we set the time between transmissions equal to the number of control signals included in each packet, $N+1$.

Lemma 1: The fixed transmission scheme with buffer length $N$ using state feedback has closed loop performance bounded by

$$
\left\|x_{k}\right\| \leq\left\|(A+B F)^{k}\right\| \chi+g(A, B, F, N, k) \bar{W}
$$

with the effect of the noise terms being accounted for in

$$
\begin{aligned}
& g(A, B, F, N, k)=\sum_{j=0}^{h(k-1, N)}\left\|A^{j}\right\| \\
& +\left(\sum_{j=0}^{\left\lfloor\left\lfloor\frac{k-1}{N+1}\right\rfloor-1\right.}\left\|(A+B F)^{s(j, k, N)}\right\|\right)\left(\sum_{j=0}^{N}\left\|A^{j}\right\|\right)
\end{aligned}
$$

where

$$
\begin{aligned}
h(k, N) & =\bmod (k, N+1) \\
s(j, k, N) & =(N+1) j+h(k-1, N)+1
\end{aligned}
$$

and \lfloor\rfloor is the floor operator.

Proof: The control packet is transmitted every $N+1$ time steps, i.e. whenever $h(k, N)=0$ the packet $\mathbf{U}_{k}$ is transmitted. This allows us to write the control applied to the plant as $u_{k}=F(A+B F)^{h(k, N)} x_{k-h(k, N)}$, and the closed loop evolution as

$$
x_{k+1}=A x_{k}+B F(A+B F)^{h(k, N)} x_{k-h(k, N)}+w_{k}
$$

It is not too difficult to use this to express the closed loop state, for $k \geq 1$, as

$$
\begin{aligned}
x_{k}=(A+ & B F)^{k} x_{0}+\sum_{j=0}^{h(k-1, N)} A^{j} w_{k-j-1} \\
& +\sum_{j=0}^{\left\lfloor\frac{k-1}{N+1}\right\rfloor-1}(A+B F)^{s(j, k, N)} d(j, k, N)
\end{aligned}
$$

where

$$
d(j, k, N)=\sum_{i=0}^{N} A^{i} w_{k-s(j, k, N)-i-1} .
$$

The first term in Eqn. (8) accounts for the initial condition. The second term is from the noise acting on the system in the time since the last transmitted control sequence, this noise cannot be compensated for by the current control sequence. The last term accounts for all the noise prior to the last transmitted packet, this is compensated for by the current control sequence through the use of $x_{k-h(k, N)}$. From Eqn. (8) and the properties of the norm and bound on $\left\|w_{k}\right\|$ we arrive at the upperbound on $\left\|x_{k}\right\|$ in Eqn. (5).

Note that whenever a packet is transmitted the control sequence is a function of the initial condition and the previous noise sequences and will only be able to compensate for those terms. For example, consider the control packet that is sent at time $k-N, \mathbf{U}_{k-N}$. The control signals from this packet are applied between time $k-N$ and time $k$ and will compensate for the noise sequence prior to time $k-N$ which are manifest in $x_{k-N}$ and hence $\mathcal{I}_{k-N}$. The noise terms $\left\{w_{k-N+1}, \ldots, w_{k}\right\}$ will not be compensated by the control sequence and their effect can be amplified by the open loop dynamics, this is the second term on the right hand side of Eqn. (8).

The fixed transmit scheme is simple to implement and it is easy to see that as the buffer length, and hence transmission interval, is increased the transmission frequency will decrease but the bound on the closed loop error will increase. This gives a design tradeoff as desired. The potential downside with the fixed communication scheme is that it might not be utilizing the network very efficiently. The control packets are transmitted at fixed points in time regardless if their transmission will have a significant impact on the closed loop error. We seek a scheme that can chose whether or not to transmit online and utilize the network resources more efficiently.

\section{B. Input Difference Transmission Scheme (IDTS)}

We propose a simple algorithm that at each time step will determine if the control packet should be sent. The algorithm computes a sequence of controls at every time step but will only transmit this sequence to the plant if the difference between the newly computed control sequence and the last sequence sent to the plant is larger than a certain threshold. This threshold becomes a design parameter and we will show its impact on the closed loop performance.

To formalize the scheme, at time $k+M$ the computed sequence of commands is $\mathbf{U}_{k+M}$ and let the last packet sent to the plant be the one sent at time $k, \mathbf{U}_{k}$. The criterion that determines whether or not to transmit packet $\mathbf{U}_{k+M}$ is based on the norm of the difference between the two control signals. Define

$\Delta U_{k}^{k+M}(j)=U_{k+M}(j)-\left\{\begin{array}{l}U_{k}(M+j) \text { if } M+j \leq N+1 \\ \gamma U_{k}(N+1) \text { otherwise }\end{array}\right.$

for $j=1, \ldots, N$. Let $\alpha_{j} \geq 0$ be a scaling factor and define the weighted norm to be

$$
\left\|\Delta U_{k}^{k+M}\right\|_{\left(\infty, \alpha_{j}\right)}=\max _{j} \alpha_{j}\left\|\Delta U_{k}^{k+M}(j)\right\| .
$$

Next pick a scalar $\bar{U}$ so the controller will only transmit the packet $\mathbf{U}_{k+M}$ to the plant if

$$
\left\|\Delta U_{k}^{k+M}\right\|_{\left(\infty, \alpha_{j}\right)}>\bar{U} .
$$

Remark 2: The 1-norm can easily replace the $\infty$-norm with slight modification to the results below. 
Based on this scheme the controller will transmit if the newly computed control command differs from the command sequence currently in the plant's buffer. In essence this will only utilize the network resources when the transmission of a control packet will have an impact on the system compared to not sending the packet. Additionally, this scheme can be combined with a force send feature, which will force transmission if the buffer at the plant has been exhausted, i.e. the time since the last transmit is greater than the length of the control sequence. The $\alpha_{j}$ coefficients and $\bar{U}$ value are also available design choices. The new transmission scheme is illustrated in Table I.

TABLE I

INPUT DifFERENCE TRANSMISSION SCHEME (IDTS).

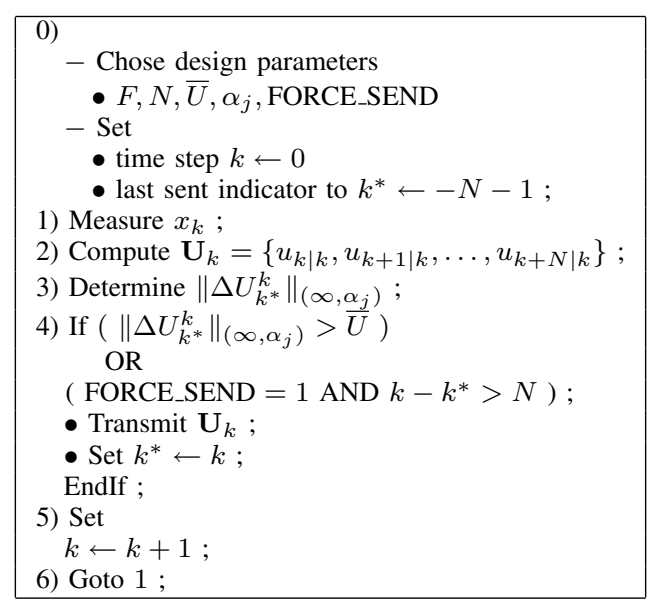

Lemma 3: With the IDTS using the state feedback controller so that $\mathbf{U}_{k}$ is given by Eqn. (4) then if $\alpha_{1}=1$, the norm of the state is bounded according to

$\left\|x_{k}\right\| \leq\left\|(A+B F)^{k}\right\| \chi+\sum_{j=0}^{k-1}\left\|(A+B F)^{j}\right\|(\|B\| \bar{U}+\bar{W})$.

Proof: Consider that at time $k$ the last packet transmitted was at time $k-M$. The control applied to the plant at time $k$ can be written as

$$
u_{k}=u_{k \mid k}-\left(1-\lambda_{k}\right) \cdot \Delta U_{k-M}^{k}(1)
$$

where $\lambda_{k} \in\{0,1\}$ is used to indicate if the control packet $\mathbf{U}_{k}$ is transmitted or not.

Since we are using a state feedback controller, we have $u_{k \mid k}=F x_{k}$ and we can write Eqn. (13) as

$$
\begin{aligned}
& u_{k}=F x_{k}+z_{k} \\
& z_{k}=\left(1-\lambda_{k}\right) \cdot \Delta U_{k-M}^{k}(1) .
\end{aligned}
$$

If $\lambda_{k}=1$ then $\mathbf{U}_{k}$ is transmitted and we have $z_{k}=0$. If $\lambda_{k}=0$ then the packet is not transmitted, however, using IDTS with $\alpha_{1}=1$ if the packet is not transmitted then we are guaranteed to have $\left\|z_{k}\right\|=\left\|\Delta U_{k-M}^{k}(1)\right\| \leq \bar{U}$. Thus regardless of the value of $\lambda_{k}$, i.e. independent of the transmission status of $\mathbf{U}_{k}$, we get that $\left\|z_{k}\right\| \leq \bar{U}$.
The closed loop system can be rewritten as

$$
\begin{aligned}
x_{k+1} & =A x_{k}+B u_{k}+w_{k} \\
& =(A+B F) x_{k}+\left(B z_{k}+w_{k}\right)
\end{aligned}
$$

which is a stable system with a bounded disturbance term $\left\|B z_{k}+w_{k}\right\| \leq\|B\| \bar{U}+\bar{W}$. The state can be written as

$$
x_{k}=(A+B F)^{k} x_{0}+\sum_{j=0}^{k-1}(A+B F)^{k-j-1}\left(B z_{j}+w_{j}\right)
$$

from which the bound in Eqn. (12) directly follows.

Remark 4: The bound in Eqn. (12) is independent of the buffer length $N$. It is also a worst case analysis, but it allows comparison with the worst case analysis for the fixed transmission scheme. These worst case bounds will be conservative due to the use of the norm properties as well as assuming the worst case noise in each step, nonetheless they can be useful guides.

In addition to computing this upper bounds on the state error we would also like to characterize the transmission frequency using IDTS since the number of time steps between transmissions will no longer be fixed. In Lemma 3 the condition $\alpha_{1}=1$ was imposed. In fact if we set $\alpha_{j}=0 \forall j \geq 2$ the result is unaffected and the transmit criterion in Eqn. (11) becomes

$$
\left\|\Delta U_{k}^{k+M}(1)\right\|>\bar{U} \text {. }
$$

This means the decision to transmit the control packet depends only on the difference between the control signal for the current time step and not the future control signals, though they will be transmitted in the packet. Since the condition to transmit is checked at every time step, removing the dependence on the future control signals is less critical and will simplify the analysis. Likewise we will assume a zero control scheme if the buffer runs out, i.e. $\gamma=0$, which will also simplify the analysis below. Thus for the remainder of this section we will assume

- $\alpha_{1}=1$ and $\alpha_{j}=0$ for $j \geq 2$

- $\gamma=0$

though similar results can be obtained without these.

In the analysis below we will make use of the following quantity. With $m \geq 1$ a positive integer define

$$
\begin{aligned}
& L(m, k, N)=\sum_{j=0}^{m-1} A^{m-j-1} w_{k+j} \\
& \quad+\delta(m-N-1) A^{m-N-1}(A+B F)^{N+1} x_{k}
\end{aligned}
$$

with

$$
\delta(j)=\left\{\begin{array}{l}
0, \text { if } j \leq 0 \\
1, \text { if } j>0
\end{array} .\right.
$$

Proposition 5: Given the last transmission occurred at time $k$, the next transmission will be at time $k+M$ where

$$
M=\min \left[\frac{N+1}{\beta}, \min _{m>0}\{m:\|F \cdot L(m, k, N)\|>\bar{U}\}\right],
$$


and $\beta$ is used to indicate if using the force send feature $(\beta=1)$ or $\operatorname{not}(\beta=0)$.

Proof: The packet transmitted at time $k$ is given by Eqn. (4). We are interested in the time when the next packet is sent, so we can write the closed loop evolution based on no packet being sent between $k$ and $k+m$. For $1 \leq m \leq N+1$ the applied control signal is $u_{k+m-1}=F(A+B F)^{m-1} x_{k}$ and the state is given by

$$
x_{k+m}=(A+B F)^{m} x_{k}+\sum_{j=0}^{m-1} A^{m-j-1} w_{k+j} .
$$

No control will be applied starting at time $k+N+1$, i.e. $u_{k+i}=0$ for $i=N+1, N+2, \ldots, m-1$, thus for $m>N+1$ the state can be written as

$$
x_{k+m}=A^{m-N-1}(A+B F)^{N+1} x_{k}+\sum_{j=0}^{m-1} A^{m-j-1} w_{k+j}
$$

From this and the fact that $u_{k+M \mid k+M}=F x_{k+M}$, it is easy to see that $\left\|\Delta U_{k}^{k+m}(1)\right\|=\|F \cdot L(m, k, N)\|$. The next transmission will occur at the first instance that $\left\|\Delta U_{k}^{k+m}(1)\right\|>\bar{U}$, or if force send is in effect it will occur at time $k+N+1$ if $m>N+1$. This is exactly the expression captured in Eqn. (15).

With the IDTS using the force send feature and state feedback control, the number of time steps between successive transmissions is simply a function of the realization of the noise sequence and the buffer length, it is independent of the state. That is to say with the force send feature it is not possible to wait longer than $N+1$ time steps so we only need to evaluate $\|F \cdot L(m, k, N)\|$ for $m \leq N+1$ and from Eqn. (14) we see the $x_{k}$ term disappears so it is only a function of the open loop dynamics and realization of the noise sequence from time $k$. Without the force send feature it is possible to wait longer than $N+1$ time steps so we check all $m \geq 1$ and for $m>N+1$ and the state $x_{k}$ will appear in the expression for $L(m, k, N)$ meaning in this case the number of time steps between transmissions depends on the value of the state at the last transmission.

As can be seen in Proposition 5 the design parameter $\bar{U}$ affects the time between transmissions. In fact, it is possible to show how the minimum time between transmissions depends on $\bar{U}$ and $\bar{W}$.

\section{Lemma 6: Define}

$$
m^{*}=\min _{m>0}\left\{m:\|F\| \cdot \bar{W} \cdot \sum_{j=0}^{m-1}\left\|A^{j}\right\|>\bar{U}\right\} .
$$

Then using IDTS with state feedback control a lower bound on the time between transmissions is given by

$$
M^{*}=\min \left[N+1, m^{*}\right] \text {. }
$$

Proof: As seen in Proposition 5 the key in determining the time between transmissions is the term

$$
\|F \cdot L(m, k, N)\|>\bar{U} .
$$

For $m \leq N+1$ the second term in Eqn. (14) drops out and we can write

$$
\begin{aligned}
\|F \cdot L(m, k, N)\| & =\left\|F \cdot \sum_{j=0}^{m-1} A^{m-j-1} w_{k+j}\right\| \\
& \leq\|F\| \cdot \bar{W} \cdot \sum_{j=0}^{m-1}\left\|A^{j}\right\| .
\end{aligned}
$$

Thus $\|F\| \cdot \bar{W} \cdot \sum_{j=0}^{m-1}\left\|A^{j}\right\|>\bar{U} \quad$ is a necessary condition for $\|F \cdot L(m, k, N)\|>\bar{U}$, i.e. it will require at least $m^{*}$ time steps before the noise alone could trigger the transmit criterion. If $m^{*}>N+1$ then the value of the state $x_{k}$ will affect the value of $\left\|\Delta U_{k}^{k+m^{*}}\right\|$, however, with force send the packet will automatically be sent after $N+1$ time steps, and without force send the lower bound of $N+1$ will still hold. Hence we arrive at Eqn. (17).

Remark 7: With force send the transmission time will be in the interval $\left[M^{*}, N+1\right]$ and without force send it will be in the interval $\left[M^{*}, \infty\right)$. Thus if $M^{*}=N+1$, meaning $m^{*} \geq N+1$, then with force send the time between transmissions will be exactly fixed at $N+1$, i.e. it recovers the fixed transmission scheme.

Remark 8: When $N+1>>M^{*}$ the transmission rate is qualitatively the same whether or not force send is used. This behavior is expected since the only difference is that without force send the transmission time can lie in the interval $[N+$ $2, \infty)$. With $N+1>>M^{*}$, however, this will rarely occur and it will more likely be in $\left[M^{*}, N+1\right]$ for both schemes.

\section{EXAMPLES}

We consider the plant in Eqn. (1) with

$$
A=\left[\begin{array}{rrrr}
1.2 & 0.4 & 1.2 & 1.5 \\
-0.2 & -0.4 & -0.2 & -0.4 \\
0.1 & -0.2 & 1.6 & 2.0 \\
-0.2 & 0.4 & 1.1 & 1.3
\end{array}\right], B=\left[\begin{array}{ll}
0 & 1 \\
0 & 0 \\
0 & 1 \\
1 & 0
\end{array}\right]
$$

This has open loop eigenvalues of $[2.79,1.25,0.37,0.092]$. The disturbance was bounded according to $\bar{W}=2$. The state feedback gain was chosen to place the closed loop eigenvalues at $[0.267,0.234,0.15,0.12]$. A total of 10,000 simulations of 100 time steps each were used to generate the noise sequences and initial conditions. The closed loop system was then simulated with the different communication schemes and various buffer lengths.

We used buffer lengths of $N=$ $\{0,1,2,3,4,5,6,7,8,9,10,20\}$ and bounds of $\bar{U}=\{20,500\}$. With $\bar{U}=20$ we have $m^{*}=2$, i.e. without force send the IDTS will always skip at least 1 time step in between transmissions and with $\bar{U}=500$ we have $m^{*}=5$. In Fig. 2 we plot the transmit properties. The top plot shows the percent of time the control packet is transmitted to the plant. The transmit rate is the same for IDTS with force send and the fixed transmission scheme 
when $N+1<m^{*}$. For IDTS, as $N>>M^{*}$ we see that the percent of time transmitting is roughly the same with or without force send and only depends on $\bar{U}$. As the transmit criterion $\bar{U}$ is increased the controller does not transmit as often. The bottom plot is the percent of time no control is applied and the plant evolves completely open loop, i.e. $u_{k}=0$. This will occur only when the controller does not transmit a packet and the time since the last transmit is greater than $N+1$. This can not happen with the force send so we only plot the cases without force send.
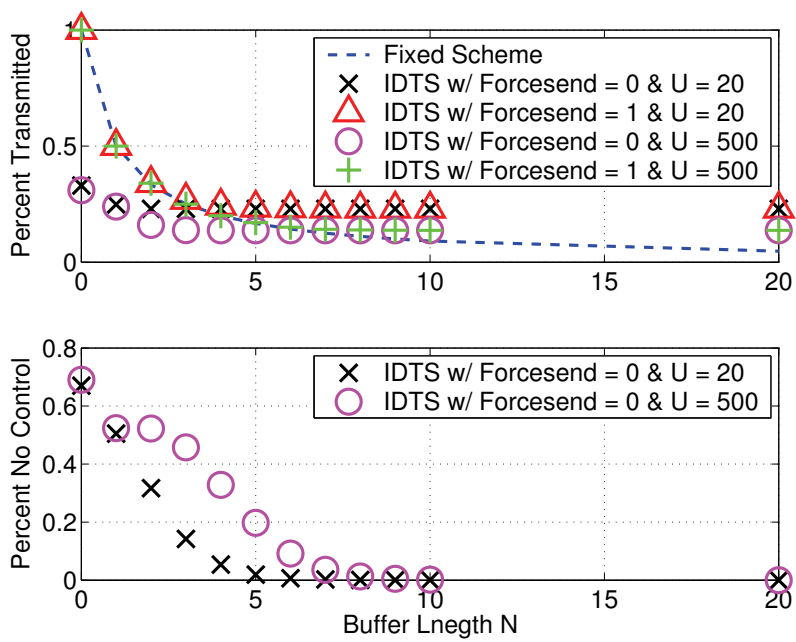

Fig. 2. Simulation results. Top plot shows the percent of time the controller transmits to the plant. Bottom plot is the percent of time the plant evolves without applying any control, i.e. $u_{k}=0$.

In Fig. 3 the state errors are plotted. The maximum error over all simulation time steps and the theoretical upper bounds are plotted. Again notice that for $N>>M^{*}$ the IDTS with and without force send exhibit the same closed loop error characteristics. Notice that for very similar transmit rates, the IDTS has smaller error compared to the fixed transmission scheme. This is evidence that the IDTS makes more effective use of the network by transmitting the packets when it is deemed important.

\section{CONCLUSIONS AND FUTURE WORK}

We considered an NCS setting where the control signal is sent across a network to the plant. The goal was to design a system that sent less frequent but more informative information packets. The data in each control packet contains the control signal for the current time step as well as a buffered sequence of predicted future control signals.

While this initial study introduced the IDTS communication scheme to reduce the transmission frequency and provided some initial insights into the performance characteristics, there is certainly more work that can be done. From the modeling standpoint, one can consider the effect of measurements taken from noisy sensors and using an observer. Network effects such as lost packets, delays and quantization were all ignored, it would be interesting to see how the communication schemes presented here would work

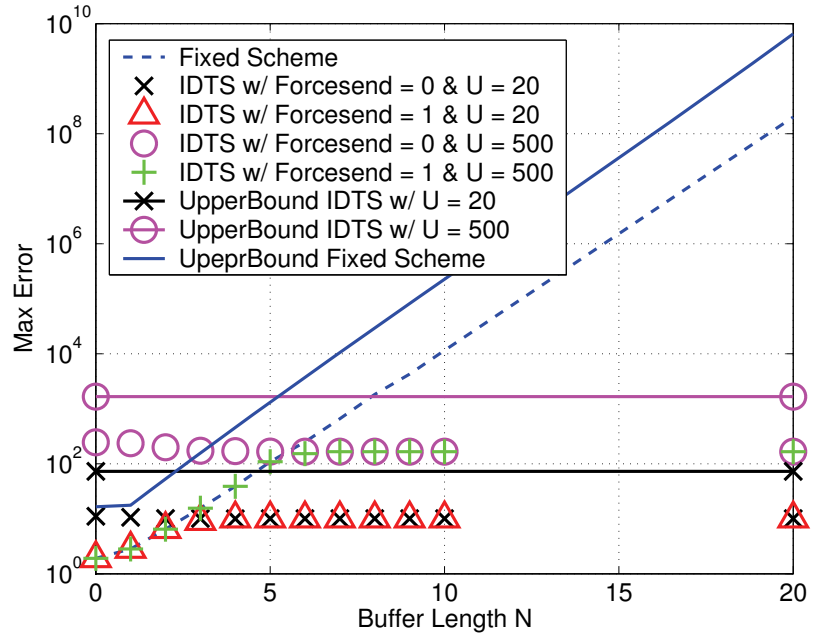

Fig. 3. Worst case simulation error and theoretical bounds. The IDTS bound is given by Eqn. (12), while the fixed transmission bound that is plotted is the $\max _{k}$ of the equation given in Eqn. (5).

when these scenarios are present and what modifications could be made to compensate for these effects.

In this work it was assumed the state feedback controller, $F$, was designed without regard to the network considerations. The closed loop properties will depend on the gain, for example a smaller $\|F\|$ will increase the minimum number of steps between transmissions. This relationship could be investigated in further detail. It would also be interesting to consider more general Model Predictive Controllers in place of the anticipative controller.

\section{REFERENCES}

[1] W. Zhang, M. Branicky, and S. Phillips, "Stability of networked control systems," vol. 21, no. 1, pp. 84-99.

[2] P. Antsaklis and J. Baillieul, "Special issue on networked control systems," 2004, vol. 49, no. 9.

[3] F. Lian, F. Moyne, and D. Tillbury, "Performance evaluation of control networks," IEEE Control Systems Magazine, vol. 21, pp. 66-83, 2001.

[4] Z. Jin, S. Waydo, E. Wildanger, M. Lammers, H. Scholze, P. Foley, D. Held, and R. M. Murray, "Mvwt-ii: The second generation caltech multi-vehicle wireless testbed." American Control Conference, 2004.

[5] L. Zhang and D. Hristu-Varsakelis, "Communication and control codesign for networked control systems," Automatica, vol. 42, 2006.

[6] D. Nesic and A. R. Teel, "Input-output stability properties of networked control systems," Automatica, vol. 49, 2004.

[7] Y. Xu and J. P. Hespanha, "Optimal communication logics in networked control systems," in Intl. Conf. on Decision and Control, 2004.

[8] L. Shi, M. Epstein, and R. M. Murray, "Towards robust control over a packet dropping network," in Mathematical Theory of Networks and Systems, 2006.

[9] C. Robinson, G. Baliga, S. Graham, and P. Kumar, "Design patterns for robust and evolvable networked control," in Conference on Systems Engineering Research, 2005.

[10] D. Georgieve and D. M. Tillbury, "Packet-based control: The $h_{2^{-}}$ optimal solution," Automaitca, vol. 42, pp. 137-144, 2006.

[11] P. A. Kawka and A. G. Alleyne, "Stability and performance of packetbased feedback control over a markov channel," in American Control Conference, 2006.

[12] P. Naghshtabrizi and J. Hespanha, "Anticipative and non-anticipative controller design for network control systems," in Networked Embedded Sensing and Control. Springer, 2006, pp. 203-218. 